(c) 2020 Universidad Nacional Autónoma de México, Facultad de Estudios Superiores Zaragoza.

Este es un artículo Open Access bajo la licencia CC BY-NC-ND (http://creativecommons.org/licenses/by-nc-nd/4.0/).

TIP Revista Especializada en Ciencias Químico-Biológicas, 23: 1-11, 2020.

https://doi.org/10.22201/fesz.23958723e.2020.0.255

\title{
Impacto de las tecnologías de extracción verdes para la obtención de compuestos bioactivos de los residuos de frutos cítricos
}

\author{
Jorge Enrique Wong-Paz, Pedro Aguilar-Zárate, \\ Fabiola Veana y Diana Beatriz Muñiz-Márquez* \\ Departamento de Ingenierías, Tecnológico Nacional de México, Campus \\ Instituto Tecnológico de Ciudad Valles, Carretera al Ingenio Plan de Ayala \\ Km. 2, Col. Vista Hermosa, Ciudad Valles, 79010, San Luis Potosí, México. \\ E-mail:*diana.marquez@tecvalles.mx
}

\begin{abstract}
RESUMEN
En la actualidad, las técnicas de extracción convencionales como la maceración, soxhlet y el calentamiento-reflujo entre otras, han seguido utilizándose debido a su principal ventaja como procesos de extracción más económicos. Sin embargo, estas metodologías presentan diversas desventajas, como largos tiempos de extracción y requerimiento de altas cantidades de disolventes. Es por lo hasta aquí expuesto que se han implementado metodologías de extracción alternativas que cumplen con los principios de la química verde, como son las extracciones asistidas: por ultrasonido(EAU), microondas (EAM) y con fluidos supercríticos (EAFS). Estos métodos de extracción, alternos a los convencionales, han logrado despertar el interés de los investigadores como futuras perspectivas de aplicación, con altos rendimientos, para la recuperación de compuestos bioactivos de los residuos de frutos cítricos, en menor tiempo de extracción, empleando disolventes verdes. El objetivo de la presente revisión es dar a conocer el impacto de las metodologías de extracción alternativas con un enfoque en el aprovechamiento y revalorización de los residuos de frutos cítricos, debido a que éstos presentan una gran diversidad de compuestos de interés para la industria farmacéutica, alimentaria y biotecnológica. Palabras clave: tecnologías de extracción verdes, residuos de frutos cítricos, compuestos bioactivos, ultrasonido, microondas.
\end{abstract}

Impact of green extraction technologies to obtain bioactive compounds from citrus fruit residues

\begin{abstract}
Today, the conventional extraction techniques as maceration, soxhlet, heat-reflux, in among others, have continued to be used because the main advantage is that are inexpensive extraction processes. However, these methodologies present various disadvantages, as long extraction times and they require high amounts of solvents. It is because of what has been stated so far that alternative extraction methodologies that comply with the principles of green chemistry have been implemented, as assisted extractions: by ultrasound (UAE), microwave (MAE) and supercritical fluids (SFAE). These extraction methods alternative to the conventional methods have managed to arouse the interest of researchers with future perspectives of application for the recovery of bioactive compounds from citrus fruit residues in less extraction time using green solvents. The objective of this review is to present the impact of alternative extraction methodologies with a focus on the use and revaluation of citrus residues because these present a great diversity of compounds of interest for the pharmaceutical, food and biotechnological industries.
\end{abstract}

Keywords: Green extraction technologies, citrus fruit residues, bioactive compounds, ultrasound, microwaves.

Artículo recibido el 23 de marzo del 2020.

Artículo aceptado el 02 de octubre del 2020. 


\section{INTRODUCCIÓN}

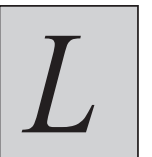

os cítricos representan la mayor demanda en la industria de los alimentos, principalmente bebidas, debido a su alto contenido de jugo para elaborar productos como néctares o zumos (Kim \& Kim, 2016). Entre las variedades de cítricos empleados se encuentran las naranjas (Citrus sinensis), mandarinas (C.unshi, C.tangerine, $C$. reticulate, $C$. clementine), limones $(C$. limon), limas $(C$. aurantifolia/latifolia), toronjas $(C$. paradisi) y pomelos $(C$. grandis) (Chavan, Singh \& Kaur, 2018). Sin embargo, una vez que son procesados se genera una gran cantidad de residuos orgánicos formados por la parte no comestible de la fruta, como lo son la cáscara (flavedo y albedo) y las semillas. Éstos representan alrededor del 50-60\% del total de la fruta (Mahato, Sharma, Sinha \& Cho, 2020). Los residuos orgánicos ocasionan un serio problema de contaminación al medio ambiente y las opciones para su tratamiento son escasas debido a la falta de conocimiento y de infraestructura para su manejo correcto (Mahato et al., 2020). No obstante, una de las opciones para su aprovechamiento ha sido el interés por su contenido en compuestos bioactivos, ya que las cáscaras son ricas en vitaminas y compuestos antioxidantes como los carotenoides, polifenoles, flavonoides, entre otros (Selvamuthukumaran \& Shi, 2017). Estas moléculas, reconocidas como metabolitos secundarios de las plantas, son extraídas comúnmente por técnicas convencionales como la maceración, hidrodestilación, soxhlet y el calentamiento-reflujo (Soquetta, Terra \& Bastos, 2018); pero a pesar de ser técnicas eficientes, se corre el riesgo de degradar los compuestos de interés por el uso de altas temperaturas y tiempos prolongados de extracción (Khan, Abert-vian, Fabiano-Tixier, Dangles \& Chemat, 2010).

Los esfuerzos para evitar las desventajas que presentan los métodos de extracción convencionales, han dado como resultado la implementación de otras técnicas conocidas como "tecnologías verdes" que además de que presentan diversas ventajas en el proceso de extracción, no impactan negativamente al medio ambiente, debido a que permiten reducir significativamente el uso de disolventes orgánicos o bien, emplear disolventes verdes. Por otro lado, mejoran el rendimiento y la calidad de los compuestos bioactivos obtenidos a partir de los residuos de frutos cítricos (Dar, Hussain, Paracha \& Akhter, 2015). Las principales técnicas amigables con el medio ambiente son las extracciones asistidas por ultrasonido (EAU), microondas (EAM), líquidos presurizados (ELP) y fluidos supercríticos (Carciochi et al., 2017); siendo las más utilizadas la EAU y la EAM debido a que se han reportado altos rendimientos en la obtención de compuestos bioactivos con menor cantidad de disolvente, en tiempos cortos de extracción (Bandar et al., 2013). Sin embargo, a pesar de contar con estas alternativas, son pocas las investigaciones que utilizan estas técnicas para extraer y recuperar compuestos bioactivos a partir de los residuos de frutos cítricos (Khan et al., 2010; Boudhrioua, M'hiri, Ioannou, Paris \& Ghoul, 2016; Safdar et al., 2017).
Por lo tanto, el objetivo de la presente revisión es dar a conocer el impacto de las tecnologías de extracción verdes, haciendo especial énfasis en la extracción asistida por ultrasonido (EAU) y microondas (EAM) para la recuperación de compuestos bioactivos, enfocada al aprovechamiento y revalorización de los residuos de frutos cítricos.

\section{Aplicación aCtual de los ReSiduos de frutos CÍTRICOS}

La producción de cítricos excede más de 88 millones de toneladas anuales en todo el mundo, por lo que se considera uno de los cultivos de mayor importancia a nivel mundial. De ese número de toneladas, alrededor de 35 a 52 millones corresponde a los residuos de frutos cítricos generados por las industrias procesadoras de alimentos por lo que se considera un problema ambiental a nivel mundial (Mahato et al., 2020). Se llaman residuos de frutos cítricos, a aquellas partes anatómicas del fruto que, durante el proceso de extracción del jugo son desechadas al no ser comestibles y no representar ningún valor económico para la industria como: la cáscara, el segmento de las membranas, los residuos de médulas y las semillas (Mahato et al., 2018) (Figura 1). El aprovechamiento de esos desechos, aún sin explorar en su totalidad, han sido la elaboración de agentes encapsulantes, películas de embalaje y empaques biodegradables (Chavan et al., 2018). Mahato et al. (2020) mencionan que los residuos de frutos cítricos son una fuente renovable económica para la obtención de compuestos químicos con valor agregado para la industria farmacéutica y alimentaria, así como para la producción de etanol, biogás y otros combustibles. A continuación, se detallan algunas aplicaciones actuales de los residuos de estos frutos a nivel mundial:

\section{Alimento para animales}

Actualmente, las opciones para aprovechar las propiedades de los residuos son diversas: como fertilizante para plantas, alimento para animales, en la industria agropecuaria, y secos para la extracción de pectina. Sin embargo, la desventaja de realizar estas acciones es que su traslado, almacenamiento y

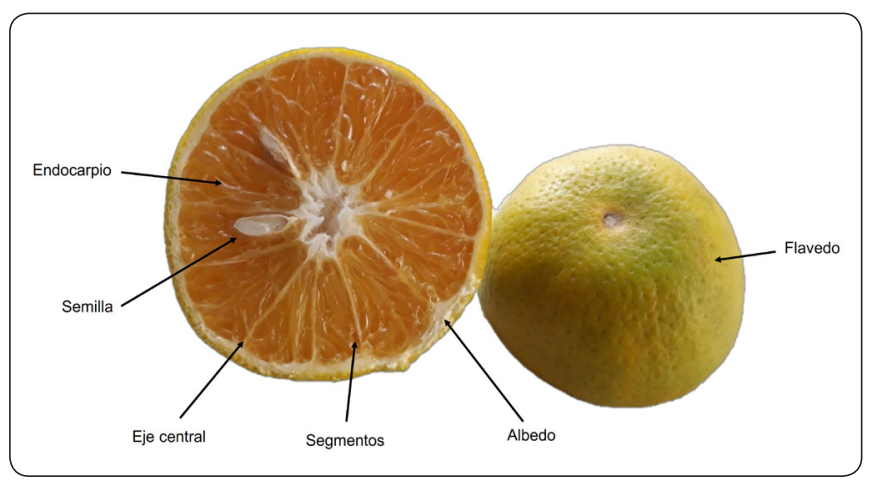

Figura 1. Estructura anatómica de los cítricos. Se observan las principales estructuras en la naranja; A) flavedo y albedo, B) semillas, C) segmentos de membranas. 
tratamiento es costoso (Vergamini, Cuming \& Viaggi, 2015). En el caso de alimento para el ganado, la pulpa de los cítricos seca es utilizada como suplemento para las vacas en etapa de lactancia, debido a que son altamente digeribles y aportan más energía que los cereales con los que son alimentados. Asimismo, la pulpa seca también puede ser incorporada como alimento para conejos, y fermentada para la alimentación de cerdos (Mahato et al., 2018).

\section{Aceites esenciales}

La extracción de aceites esenciales a partir también de los residuos mencionados es una actividad que ha sido de gran interés para la industria farmacéutica y cosmética debido a la variedad de compuestos volátiles que poseen (Chavan et al., 2018). El más estudiado es el aceite esencial del limón (C. limon) pues posee terpenoides que tienen capacidad antifúngica(Hernawan, Radhithia, Hadi \& Ernawati, 2015). No obstante, los aceites de naranja, toronja y tangerina también son importantes, sin embargo, aquellos que son obtenidos a partir del limón y la lima son los más empleados para la elaboración de perfumes, en la industria farmacéutica como aromatizantes para enmascarar sabores desagradables en los medicamentos, y en la industria alimentaria como agentes saborizantes o potenciadores de sabor de algunos productos alimentarios (Kodagoda \& Marapana, 2017).

\section{Polisacáridos (Pectina)}

Otra de las aplicaciones de los residuos es la obtención de pectina a partir de la cáscara. Su uso comercial va desde agente gelificante hasta estabilizante. La pectina es un polímero del ácido D-galacturónico ampliamente utilizado en la industria de alimentos, debido a que mejora la estabilidad de los productos alimentarios así como sus propiedades sensoriales (Chavan et al., 2018). La pectina se obtiene a partir de las cáscaras de cítricos como lima, naranja, toronja y limón, sin embargo, también se obtiene de otras fuentes como orujo de manzana o remolacha de la caña de azúcar; pero a pesar de ello, las pectinas cítricas son consideradas como unas de las más importantes (Zhao et al., 2020). Otras de las funciones de la pectina es como agente texturizante, emulsificador, fibra dietética y espesante (Putnik et al., 2017).

\section{Biocombustibles}

Es interesante saber que a partir de la biomasa producida por los cítricos se puede obtener biocombustible mediante una serie de procesos que involucran el uso de catalizadores químicos y energía térmica (Chavan et al., 2018). Esto es posible debido a que contienen cantidades considerables de carbohidratos solubles e insolubles en agua, lo que facilita la producción de biogás, así como la producción de etanol (Putnik et al., 2017). A pesar de que son pocas las investigaciones que se han realizado al respecto, esto representa una enorme ventaja para los combustibles fósiles puesto que son fuentes renovables y biodegradables, y disminuye la emisión de gases de efecto invernadero (Bull \& Obunwo, 2014). Por otro lado, algunas investigaciones han estado orientadas en el uso de aceites esenciales de cítricos para la síntesis química (metátesis) de moléculas de base biológica que pudieran ser empleadas para la obtención de energía (Martínez, Gutiérrez \& Tlenkopatchev, 2012).

\section{Adsorbentes}

La adsorción es un proceso mediante el cual se facilita la remoción de contaminantes peligrosos como metales, productos de las actividades industriales de los efluentes (Chavan et al., 2018). De ahí que, principalmente las cáscaras, son aprovechadas con la finalidad de conocer el tipo de contaminantes que pueden adsorber y por lo tanto hacer más eficiente su remoción. Las ventajas de usar estos residuos es que son de bajo costo, están disponibles en todo momento, requieren de un diseño simple, tienen buena capacidad de adsorción, generan menos problemas en su tratamiento al final de su uso, es decir, baja cantidad de subproductos después del proceso, entre otras (Pathak, Mandavgane \& Kulkarni, 2015). En este sentido, Marín-Rangel, Cortés-Martínez, Cuevas-Villanueva, Garnica-Romo \& Martínez-Flores (2012) evaluaron las cáscaras de limón (C. aurantifolia var. Swingle) tratadas con $\mathrm{FeCl}_{3}$ para la remoción de arsenato, As (V), a partir de una solución de arsenato de sodio. Los resultados demostraron la capacidad del limón en adsorber hasta $474.8 \mu \mathrm{g}$ de As/g de bioadsorbente.

\section{COMPUESTOS BIOACTIVOS DE LOS CÍTRICOS}

Los cítricos son las frutas que más se consumen alrededor del mundo, por su contenido en zumos que son una fuente importante y natural de vitamina $\mathrm{C}$, ácido fólico, potasio y compuestos fenólicos; que presentan diversas actividades biológicas, destacando la antioxidante. Los compuestos fenólicos son fitoquímicos que derivados del metabolismo secundario de las plantas, y con efecto antioxidante coadyuvan en prevenir el daño oxidativo de las células, ocasionado por especies reactivas de oxígeno (EROS), o sea moléculas relacionadas con enfermedades crónicas como cáncer, diabetes, neurodegenerativas y disfunciones cardiovasculares (Rafiq et al., 2018). Los compuestos fenólicos de los cítricos, se caracterizan por la presencia de grupos hidroxilo $(-\mathrm{OH})$ que están unidos al carbono 6 del anillo aromático y por lo tanto pueden estar como glicósidos libres o formas glicosídicas (Medina-Torres, Ayora-Talavera, Espinosa-Andrews, Sánchez.-Contreras \& Pacheco, 2017). También tienen flavonas glicosídicas, es decir, con restos de azúcar como la naringina, hesperidina y eriocitrina; además de flavonas agliconadas, sin restos de azúcar unidos a las moléculas, por ejemplo: la naringenina, hesperetina y eriodictiol (De-la-Rosa-Hernández, Wong-Paz, Muñiz-Márquez, Carrillo-Inungaray \& Sánchez-González, 2016). Los principales compuestos bioactivos de los cítricos son los flavonoides, flavonoles, antocianinas, flavonas, flavanonas, carotenoides, cumarinas así como limonoides. Entre los flavonoides, se encuentran la hesperidina, narirutina, naringina y 
eriocitrina (Nakajima, Macedo \& Macedo, 2014) (Figura 2). Es importante destacar que no todos los compuestos mencionados están presentes en todos los cítricos, sino que varían entre sí (Tabla I) e inclusive puede haber otros compuestos de interés con capacidad antioxidante diferente.

Al-juhaimi (2014) determinó el contenido de fenoles totales por el método de Folin-Ciocalteu, así como la capacidad antioxidante por el método del radical libre 2,2-difenil-1picrilhidracilo (DPPH); de la cáscara y pulpa de naranja, mandarina y limón. Sus resultados demostraron que los tres cítricos tienen buen contenido de fenoles totales: naranja (pulpa: $123.02 \mathrm{mg} \mathrm{GAE} / 100 \mathrm{~g}$; cáscara: $178.90 \mathrm{mg} \mathrm{GAE} /$ $100 \mathrm{~g}$ ), mandarina (pulpa: $104.98 \mathrm{mg} \mathrm{GAE} / 100 \mathrm{~g}$; cáscara: 169.54 mg GAE/100 g) y limón (pulpa: $98.28 \mathrm{mg} \mathrm{GAE} / 100 \mathrm{~g}$; cáscara: $61.22 \mathrm{mg}$ GAE/100 g) así como un buen porcentaje de actividad antioxidante: naranja (pulpa: $69.31 \%$; cáscara: $67.58 \%$ ), mandarina (pulpa: $62.82 \%$; cáscara: $68.57 \%$ ) y limón (pulpa: $59.60 \%$; cáscara: $46.98 \%$ ). Por otro lado, Ergüt \& Döker (2017), analizaron fenoles y flavonoides totales, así como el potencial antioxidante de residuos deshidratados de limón y naranja compuestos por la cáscara, albedo y segmentos de la pulpa. Sus resultados mostraron que la naranja presentó un mayor contenido de fenoles totales en comparación con el limón (1268.69 $\mu \mathrm{g}$ GAE/g a $1839.54 \mu \mathrm{g}$ GAE/g de material seco)y (778.75 $\mu \mathrm{g}$ GAE/g a $1164.71 \mu \mathrm{g} \mathrm{GAE} / \mathrm{g}$ de material seco) respectivamente. De igual manera, el contenido de flavonoides totales fue mayor en los extractos de naranja (3.49 mg a $4.53 \mathrm{mg}$ quercetina/g de materia seca) y esta tendencia fue similar a la actividad antioxidante de los extractos (3.067 a $2.269 \mu \mathrm{mol}$ trolox/g materia seca) para naranja y (2.003 a $2.415 \mu \mathrm{mol}$ trolox/g materia seca) para limón.

Una de las propiedades de los compuestos bioactivos que poseen los cítricos es su efecto antimicrobiano, entre ellos, los flavonoides presentan actividad antifúngica, antibacteriana y tienen capacidad antioxidante. Estos compuestos están presentes en la cáscara, en el jugo así como en las semillas (Damián-Reyna, González-Hernández \& ChávezPraga, 2016). Safdar et al. (2017), evaluaron la actividad antibacteriana de un extracto etanólico con base en la cáscara de mandarina (C. reticulate L.) contra Staphylococcus aureus, Bacillus cereus y Salmonella typhimurium, en concentraciones de $25 \mathrm{mg} / \mathrm{mL}, 50 \mathrm{mg} / \mathrm{mL}, 100 \mathrm{mg} / \mathrm{mL}$ y $200 \mathrm{mg} / \mathrm{mL}$ de extracto. Sus resultados demostraron que $S$. aureus y $B$. cereus tienen mayor sensibilidad a los compuestos bioactivos de la cáscara de la mandarina que S. typhimurium. Además, se reconoce que este efecto fue propiciado por la presencia de polifenoles en el extracto, específicamente, de flavonoles como la quercetina. Por otro lado, Madhuri, Hegde, Srilakshimi \& Prashith (2014) evaluaron la actividad antibacteriana (B. cereus, Shigella flexneri y Klebsiella pneumoniae) y antifúngica(Colletotrichum capsici) de los extractos de la cáscara de $C$. aurantium y $C$. sinensis. Los resultados obtenidos demostraron la capacidad antimicrobiana de los cítricos al inhibir notoriamente el crecimiento del hongo fitopatógeno ( $>50 \%$ de inhibición) así

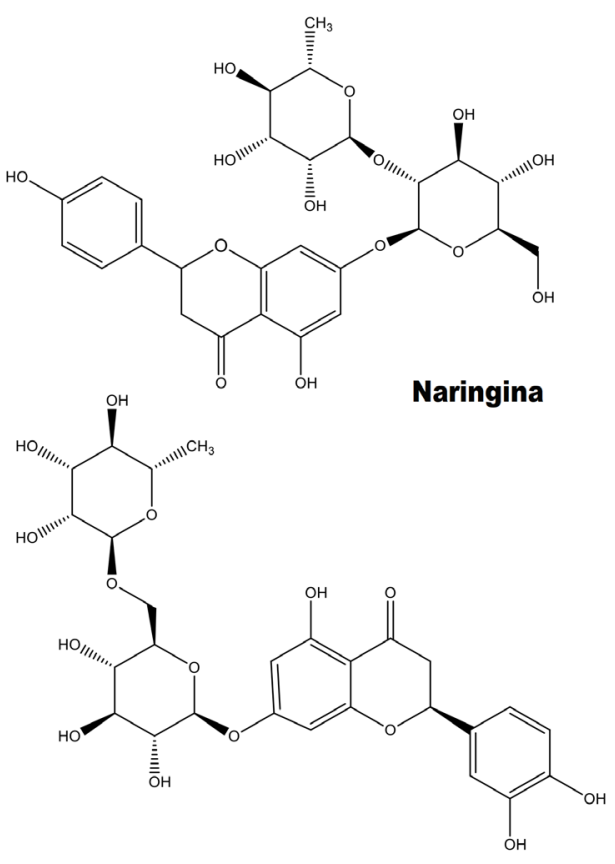

Eriocitrina

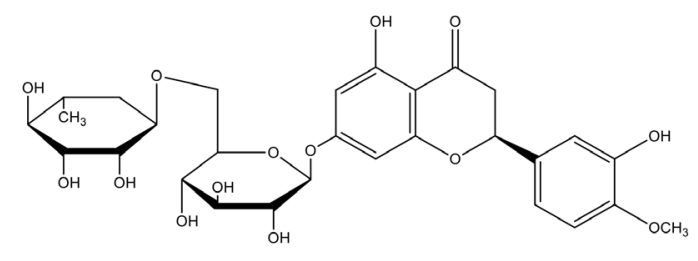

Hesperidina

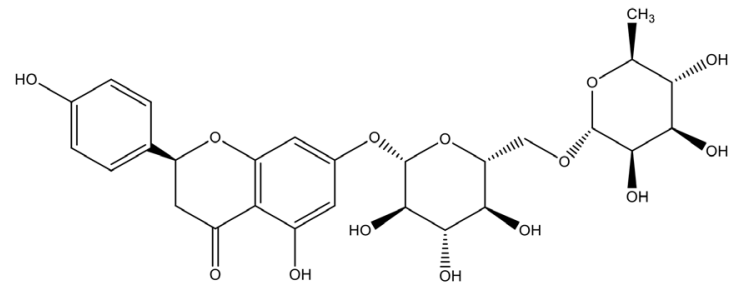

Narirutina

Figura 2. Estructuras químicas de los principales compuestos de los cítricos. Compuestos fenólicos tipo flavanonas. 
Tabla I. Principales metabolitos identificados en residuos de cítricos.

\begin{tabular}{|c|c|c|c|c|}
\hline Cítrico & Residuo & Compuestos bioactivos & Métodos de identificación/cuantificación & Referencia \\
\hline Naranja & Cáscara & $\begin{array}{l}\text { Narirutina, diosmina, } \\
\text { hesperidina, didimina, } \\
\text { sinensetina, nobiletina, } \\
\text { tangeretina }\end{array}$ & $\begin{array}{l}\text { HPLC-DAD (fase reversa, columna C- } 18 \text {, tasa } \\
\text { de flujo de } 1 \mathrm{~mL} / \text { min, gradiente de elución de } \\
\text { acetonitrilo (A) y ácido acético al } 0.6 \% \text { (B)). } \\
\text { HPLC-MS (electrospray modo positivo, voltaje } \\
\text { de capilaridad de } 3.50 \mathrm{kV} \text {, voltaje de cono } 20 \mathrm{~V} \\
\text { temperatura de } 100{ }^{\circ} \mathrm{C} \text {, intervalo de escaneo de } \\
200 \text { uma a } 800 \text { uma en modo centroide). }\end{array}$ & $\begin{array}{l}\text { (Bermejo, Llosá } \\
\text { \& Cano, 2011) }\end{array}$ \\
\hline Mandarina & Cáscara & Narirutina y hesperidina. & $\begin{array}{l}\text { HPLC (Columna Chomp SS C- } 18,282 \mathrm{~nm} \text {, tasa } \\
\text { de flujo de } 0.85 \mathrm{~mL} / \mathrm{min} \text {, gradiente de elución } \\
\text { de metanol (A) y ácido acético al } 2 \% \text { (B)) }\end{array}$ & $\begin{array}{l}\text { (Tumbas, } \\
\text { Cetkovic \& } \\
\text { Djilas, 2010) }\end{array}$ \\
\hline Toronja & Cáscara & Naringina, hesperidina, & $\begin{array}{l}\text { Electroforesis capilar con detección } \\
\text { electroquímica (sistema CE-ED). }\end{array}$ & $\begin{array}{l}\text { (Wu, Guan \& Ye, } \\
\text { 2007) }\end{array}$ \\
\hline Lima & $\begin{array}{l}\text { Bagazo } \\
\text { y orujo } \\
\text { (cáscara } \\
\text { seca) }\end{array}$ & $\begin{array}{l}\text { Naringina, hesperidina, } \\
\text { ácido cumárico, } \\
\text { catequina, pirogalol, } \\
\text { resorcinol, metil-galato, } \\
\text { quercetina, ácido elágico. }\end{array}$ & $\begin{array}{l}\text { HPLC (columna Varian C18 ODS, } 280 \mathrm{~nm} \text {, tasa } \\
\text { de flujo de } 1 \mathrm{~mL} / \mathrm{min} \text {, gradiente de elución de } \\
\text { ácido acético al } 3 \% \text { ) }\end{array}$ & $\begin{array}{l}\text { (Sánchez-Aldana, } \\
\text { Aguilar, Nevarez- } \\
\text { Moorillon } \\
\text { \& Esquivel- } \\
\text { Contreras, 2013) }\end{array}$ \\
\hline Limón & $\begin{array}{l}\text { Flavedo } \\
\text { Albedo } \\
\text { Pulpa }\end{array}$ & $\begin{array}{l}\text { Hesperidina, diosmina, } \\
\text { limetina. } \\
\text { Hesperidina, diosmina, } \\
\text { limetina. } \\
\text { Hesperidina, diosmina. }\end{array}$ & $\begin{array}{l}\text { HPLC (columna Inertsil ODS-2, } 220 \mathrm{~nm} \text {, tasa } \\
\text { de flujo de } 1 \mathrm{~mL} / \mathrm{min} \text {, gradiente de elución de } \\
\text { acetonitrilo al } 10 \% \text { (A) y acetonitrilo al } 70 \% \\
\text { (B). }\end{array}$ & $\begin{array}{l}\text { (Shie \& Lay, } \\
\text { 2013) }\end{array}$ \\
\hline
\end{tabular}

como de las bacterias. Asimismo, Ali et al. (2017) demostraron la capacidad antimicrobiana de un extracto metanólico de la cáscara del limón $(C$. limon) al inhibir considerablemente el crecimiento de $S$. aureus, E. coli, Candida albicans y de Trichophyton rubrum a una concentración mínima inhibidora de $100 \mu \mathrm{g} / \mathrm{mL}$. Mediante análisis fitoquímicos fueron identificados compuestos como los alcaloides, terpenoides, saponinas, esteroles y flavonoides.

\section{EXTRACCIÓN DE COMPUESTOS BIOACTIVOS DE LOS RESIDUOS DE FRUTOS CÍTRICOS}

La presencia de compuestos bioactivos en las plantas, principalmente de fitoquímicos como los polifenoles han despertado gran interés por las propiedades antioxidantes y antimicrobianas que presentan. Por ello, la extracción de estos compuestos ha sido de interés para las industrias alimentaria, farmacéutica e inclusive cosmética, y se realiza a partir de residuos de los cítricos, para la elaboración de productos funcionales (Wang \& Weller, 2006; Selvamuthukumaran \& Shi, 2017). Tradicionalmente, los métodos de extracción de compuestos bioactivos utilizados son maceración, hidrodestilación y Soxhlet. Sin embargo, estas técnicas utilizan grandes cantidades de disolventes químicos, involucran en algunos casos agitación, y requieren de largos periodos de tiempo para la extracción, que va desde $8 \mathrm{~h}$ para la hidrodestilación a más de 24 h para la maceración (Soquetta et al., 2018). Ante esto, y como ya se mencionó, se han estudiado nuevas formas de extracción más amigables con el medio ambiente, debido a que utilizan disolventes verdes o bien, menor cantidad de disolventes orgánicos, y requieren de menor tiempo de extracción. A estos métodos de extracción se les conoce como tecnologías emergentes o tecnologías no convencionales, siendo las de mayor importancia las siguientes extracciones: la asistida por ultrasonido(EAU), por microondas (EAM) y por fluidos supercríticos (EAFS). No obstante, las más empleadas han sido las dos primeras debido a que de acuerdo a estudios previos, estos métodos permiten incrementar los rendimientos de obtención de compuestos bioactivos, son más accesibles y fácil de operar (Wong-Paz et al., 2017), además se obtienen compuestos de buena calidad y en alto rendimiento, sin la necesidad de emplear grandes volúmenes de disolventes, al igual que el gasto energético, que es bajo debido a que se requieren tiempos muy cortos para la extracción, que pueden ser desde 5-40 min, aproximadamente (Putnik et al., 2017). En lo que respecta a los cítricos, en la Tabla II se muestran algunas técnicas convencionales y alternativas para la recuperación de compuestos de estos materiales.

\section{Extracción asistida por ultrasonido (EAU)}

La extracción asistida por ultrasonido (EAU) no es un método de extracción novedoso, ya que ha sido ampliamente utilizado en la extracción de compuestos de plantas y frutas desde los 
Tabla II. Procesos de extracción convencionales y no convencionales aplicados a residuos cítricos.

\begin{tabular}{|c|c|c|c|c|}
\hline Cítrico & $\begin{array}{c}\text { Tipo de } \\
\text { extracción }\end{array}$ & Condiciones & Rendimiento & Referencia \\
\hline \multirow[t]{3}{*}{ C. aurantium } & Maceración* & $\begin{array}{l}\text { Disolventes: etanol:agua }(1: 1) \text {, etanol y agua } \\
\text { Tiempo de extracción: } 8 \mathrm{~h} \\
\text { Temperatura: } 17^{\circ} \mathrm{C} \text { a } 20^{\circ} \mathrm{C}\end{array}$ & $\begin{array}{l}\text { Etanol/agua: } 29.28 \% \\
\text { Etanol: } 23.65 \% \\
\text { Agua: } 6.53 \%\end{array}$ & \multirow[t]{3}{*}{$\begin{array}{l}\text { (Dar et al., } \\
\text { 2015) }\end{array}$} \\
\hline & $\begin{array}{l}\text { Extracción } \\
\text { asistida por } \\
\text { ultrasonido** }\end{array}$ & $\begin{array}{l}\text { Disolventes: etanol:agua }(1: 1) \text {, etanol y agua } \\
\text { Tiempo de extracción: poder ultrasonido al } \\
50 \% \text {, } 5 \text { min y poder de ultrasonido al } 100 \% \\
\text { por } 30 \text { min. } \\
\text { Temperatura: } 25^{\circ} \mathrm{C} \mathrm{a} 30^{\circ} \mathrm{C}\end{array}$ & $\begin{array}{l}\text { Etanol/agua (50\%): } 36.92 \% ; \\
\text { Etanol/agua }(100 \%): 41.38 \% \\
\text { Etanol }(50 \%): 32.74 \% \text {; Etanol } \\
(100 \%): 40.90 \% \\
\text { Agua }(50 \%): 43.20 \% \text {; Agua } \\
(100 \%): 44.94 \%\end{array}$ & \\
\hline & $\begin{array}{l}\text { Extracción } \\
\text { asistida por } \\
\text { microondas } * *\end{array}$ & $\begin{array}{l}\text { Disolventes: etanol:agua }(1: 1) \text {, etanol y agua } \\
\text { Tiempo de extracción: } 3 \mathrm{~s} \text { a } 20 \mathrm{~s} \\
\text { Temperatura: } 80^{\circ} \mathrm{C} \text { a } 85^{\circ} \mathrm{C}\end{array}$ & $\begin{array}{l}\text { Etanol/agua: } 9.52 \% \\
\text { Etanol: } 30.21 \% \\
\text { Agua: } 22.73 \%\end{array}$ & \\
\hline $\begin{array}{l}\text { C. } \\
\text { aurantifolia } \\
\text { Swingle }\end{array}$ & $\begin{array}{l}\text { Extracción } \\
\text { asistida por } \\
\text { microondas } * *\end{array}$ & $\begin{array}{l}\text { Disolvente: } 60 \mathrm{~mL} \text { de solución al } 1 \% \text { de } \\
\text { ácido cítrico. } \\
\text { Tiempo de extracción: } 5 \mathrm{~min}, 800 \mathrm{~W} \text {. } \\
\text { Temperatura: } 120^{\circ} \mathrm{C}\end{array}$ & $\begin{array}{l}\text { Fenoles totales }=38.00 \pm 0.02- \\
41.43 \mathrm{mg} / \mathrm{g} \text { extracto }\end{array}$ & $\begin{array}{l}\text { (Sanchez- } \\
\text { Aldana et al., } \\
\text { 2013) }\end{array}$ \\
\hline $\begin{array}{l}\text { C. reticulate } \\
\text { L. }\end{array}$ & Maceración * & $\begin{array}{l}\text { Disolventes: etanol, metanol, acetona, } \\
\text { acetato de etilo }(50 \%, 80 \%, 100 \%) \\
\text { Relación material/disolvente: } 1: 15 \mathrm{w} / \mathrm{v} \\
\text { Tiempo de extracción: } 20 \mathrm{~h} \\
\text { Temperatura: } 40^{\circ} \mathrm{C}\end{array}$ & $\begin{array}{l}\text { El rendimiento más alto fue } \\
\text { utilizando etanol. } \\
80 \% \text { etanol }=18.46 \% \\
50 \% \text { etanol }=15.64 \% \\
100 \% \text { acetato de etilo }=5.12 \%\end{array}$ & $\begin{array}{l}\text { (Safdar et al., } \\
\text { 2017) }\end{array}$ \\
\hline $\begin{array}{l}\text { C. medica } \mathrm{L} \text {. } \\
\text { C. sinensis } \mathrm{L} \text {. } \\
\text { C. limon } \mathrm{L} \text {. }\end{array}$ & $\begin{array}{l}\text { Extracción } \\
\text { asistida por } \\
\text { microondas** }\end{array}$ & $\begin{array}{l}\text { Disolvente: agua } \\
\text { Potencia: } 10 \mathrm{~W} \\
\text { MAE 1: } 20 \mathrm{~min} \text { a } 80{ }^{\circ} \mathrm{C} \\
\text { MAE 2: } 8 \text { min a } 100{ }^{\circ} \mathrm{C} \\
\text { MAE 3: } 20 \mathrm{~min} \text { a } 100^{\circ} \mathrm{C}\end{array}$ & $\begin{array}{l}\text { Intervalo entre tratamientos } \\
\text { entre } 18.0 \% \text { a } 21.5 \%\end{array}$ & $\begin{array}{l}\text { (Caputo, } \\
\text { Quintieri, } \\
\text { Cavalluzzi, } \\
\text { Lentini \& } \\
\text { Habtemariam, } \\
\text { 2018) }\end{array}$ \\
\hline
\end{tabular}

*extracción convencional, **extracción no convencional.

años 80 (Wong-Paz et al., 2017). El método consiste en la propagación de ondas ultrasónicas que van desde $20 \mathrm{kHz}$ a $100 \mathrm{MHz}$. En tanto que el primer fenómeno que ocurre durante la extracción de compuestos bioactivos a partir de las plantas (hojas y cáscaras de frutas, entre otros) es la fragmentación, caracterizada por la reducción del tamaño de las partículas, lo que facilita la trasferencia de masa provocada por colisiones entre partículas y ondas propias del ultrasonido. En segundo término, la erosión participa en que el disolvente pueda llegar a la superficie de la matriz de la planta al implosionar las burbujas generadas. Por otro lado, la sonocapilaridad y sonoporación mejoran la penetración del disolvente en las membranas celulares de la planta por medio de los canales generados por la implosión de las burbujas. La liberación de los compuestos bioactivos es 
la finalidad de la última etapa, facilitada por el fenómeno de oscilación, es decir, el colapso de burbujas de cavitación al fluido producido por mecanismos de estrés (Medina-Torres, Ayora-Talavera, Espinosa-Andrews, Sánchez-Contreras \& Pacheco, 2017). Este colapso se conoce como cavitación e incluye la producción de burbujas producto del paso de las ondas ultrasónicas por el disolvente, crecimiento y colapso de las mismas en grandes cantidades (Wong-Paz et al., 2017).

Para llevar a cabo la extracción asistida por ultrasonido existen dos opciones: directamente, mediante un sistema de sondas que se sumergen en el sistema líquido-sólido con intensidades muy altas; o bien, indirectamente, con el uso de un baño de agua ultrasónico donde se coloca la muestra en un matraz con el disolvente y las ondas se transfieren a través del agua hasta el material vegetal. A partir de estas técnicas se han logrado obtener polisacáridos como pectinas, aceites esenciales, compuestos bioactivos y proteínas (Soquetta et al., 2018). A partir de las EAU se han obtenido compuestos fenólicos derivados de los residuos de maíz (Zea mays L.) como la mazorca (Hernández etal.,2018), compuestos bioactivos con potencial antioxidante a partir de Laurus nobilis L. (Lauraceae), polifenoles de Coriandrum sativum L. (Apiaceae) y Amaranthus hybridus L. (Amaranthaceae) (Muñiz-Márquez et al., 2013a), así como polifenoles de plantas del desierto mexicano como Jatropha dioica, Flourensia cernua, Turnera diffusa y Eucalyptus camaldulensis (Wong-Paz, Muñiz-Márquez, Martínez-Âvila, Belmares-Cerda \& Aguilar, 2015) y de Laurus nobilis L. (Muñiz-Márquez et al., 2013b).

En tanto que la extracción de compuestos como los polifenoles de los residuos de frutos cítricos han llamado la atención. Khan et al. (2010), realizaron la extracción de polifenoles (naringina y hesperidina) a partir de la cáscara de naranja (C. sinensis L.) por medio del diseño Box-Wilson y de la metodología de superficie de respuesta para determinar las mejores condiciones de extracción, siendo $2 \mathrm{~cm}^{2}$ de tamaño de partícula, $40{ }^{\circ} \mathrm{C}$ para temperatura, $150 \mathrm{~W}$ de poder de sonicación, a $25 \mathrm{kHz}$, utilizando un radio etanol:agua 4:1 (v/v), con un tiempo de extracción de $30 \mathrm{~min}$; logrando obtener un rendimiento de $10.9 \%$. Por otro lado, Dahmoune et al. (2014) optimizaron las condiciones de extracción para la obtención de compuestos fenólicos de cáscaras de C. sinensis L. mediante la metodología de superficie de respuesta, así como un diseño Box-Wilson. Las condiciones óptimas obtenidas fueron 8.33 min de tiempo de extracción, $75.79 \%$ de concentración de acetona acuosa y $65.94 \%$ de amplitud, con un rendimiento de $13.57 \pm 0.71 \mathrm{mg}$ $\mathrm{GAE} / \mathrm{g}$ peso seco. Las condiciones de operación mencionadas son $20 \mathrm{kHz}$ a $27 \pm 2{ }^{\circ} \mathrm{C}$. Además, Londoño-Londoño et al. (2010) evaluaron el rendimiento de los flavonoides a partir de cáscaras de lima (C. latifolia), naranja (C. sinensis) y tanjarina (C. reticulata). Las condiciones para su obtención fueron $60 \mathrm{kHz}, 30 \mathrm{~min}$ de extracción a $40{ }^{\circ} \mathrm{C}$, a un radio cáscara:agua (1:10). El rendimiento fue de $40.25 \pm 12.09$ mg de fracción flavonoide/g de cáscara, con un contenido fenólico total en fracciones flavonoides de cada cítrico de $74.80 \pm 1.90 \mathrm{mg} \mathrm{GAE} / \mathrm{g}$ de material seco para la lima, 66.36 $\pm 0.75 \mathrm{mg} \mathrm{GAE} / \mathrm{g}$ de material seco para naranja y $58.68 \pm$ $4.01 \mathrm{mg} \mathrm{GAE} / \mathrm{g}$ de material seco para tanjarina.

\section{Extracción asistida por microondas (EAM)}

La extracción asistida por microondas se considera un método alternativo a los convencionales para la obtención de compuestos bioactivos de las plantas. Las ventajas que posee son similares a la EAU: reduce el consumo de energía, la extracción requiere de menor tiempo, el gasto de disolventes es menor y genera mejor rendimiento de los compuestos de interés (Sadeghi, Hakimzadeh \& Karimifar, 2017). La frecuencia de las microondas oscila entre $300 \mathrm{MHz}$ a $300 \mathrm{GHz}$, son de naturaleza electromagnética no ionizante (Al-Mamoori \& Al-Janabi, 2018) y presentan dos campos de oscilación perpendiculares, es decir, frecuencias magnéticas y eléctricas. A partir de esta técnica de extracción se pueden obtener compuestos bioactivos, pigmentos y polisacáridos entre otros (Soquetta et al., 2018). Las formas de extraer compuestos a partir de microondas son dos: extracción por disolvente asistida por microondas, donde la muestra cubierta por el disolvente se irradia con microondas y la extracción con recipiente cerrado/multimodo utilizando alta presión y temperatura (Al-Mamoori \& Al-Janabi, 2018). De manera general se conocen tres procesos importantes para la extracción de compuestos por microondas: el primero de ellos es la separación de solutos mediante temperatura y presión elevadas, por consiguiente, ocurre la difusión del disolvente a través de la muestra y por último, la liberación de compuestos al disolvente a partir de la muestra evaluada (Sadeghi et al., 2017). La generación de ondas electromagnéticas da la pauta para el inicio del proceso de extracción permitiendo que las paredes celulares de las plantas y/o sus residuos interactúen con las ondas, dando como resultado un calentamiento en la matriz vegetal, lo que ocasiona una hinchazón en sus paredes celulares debido a la presión ejercida, provocando un incremento en la transferencia de masa de los solutos. Por lo tanto, la disrupción de las células es inevitable, liberándose los compuestos hacia el disolvente (Ameer, Shahbaz \& Kwon, 2017).

En lo que respecta a la extracción de compuestos bioactivos como los polifenoles de los residuos de frutos cítricos, los estudios que se han llevado a cabo son pocos (Tabla II). Sin embargo, algunas investigaciones se han realizado con la finalidad de optimizar la extracción de compuestos antioxidantes a partir de plantas de uso medicinal (Tsatsop, Djiobie, Kenmogne, Regonne \& Ngassoum, 2016). En lo que respecta a los residuos de las frutas, Alias \& Abbas (2017) evaluaron la extracción asistida por microondas y soxhlet de la cáscara de la piña (Ananas comosus L. Merr) para la extracción de compuestos fenólicos, utilizando $750 \mathrm{~W}$ de potencia, a una temperatura de $60^{\circ} \mathrm{C}$, por $20 \mathrm{~min}$, y una relación etanol: agua de 50:50 para el caso de microondas, y $50 \mathrm{~mL}$ de etanol en 
$200 \mathrm{~mL}$ de agua desionizada, por $5 \mathrm{~h}$, a $78^{\circ} \mathrm{C}$, para el método de soxhlet. Comprobaron que la EAM da mejores rendimientos que el método convencional. Por otro lado, Nayak et al. (2015) evaluaron la extracción de polifenoles de la cáscara de Citrus sinensis por EAM alcanzando un rendimiento máximo de compuestos bajo las siguientes condiciones: acetona acuosa al $51 \% \mathrm{v} / \mathrm{v}, 122 \mathrm{~s}$ de tiempo de extracción, $500 \mathrm{~W}$ de potencia y una relación sólido-líquido de $25 \mathrm{~mL} \mathrm{~g}^{-1}$.

\section{Extracción asistida con fluidos supercríticos (EAFS)}

La EAFS es otra de las tecnologías verdes innovadoras que puede ser usada para la obtención de compuestos bioactivos de los residuos de cítricos. Esta técnica favorece una rápida extracción utilizando temperaturas moderadas lo que impide la degradación de los compuestos de interés, elimina etapas de lavado y evita el uso de disolventes tóxicos en el proceso. El dióxido de carbono $\left(\mathrm{CO}_{2}\right)$ es el gas comúnmente empleado en la EAFS, debido a que presenta diversas ventajas por ser un gas no explosivo, moderadamente no inflamable, económico y accesible, de baja temperatura crítica $\left(32^{\circ} \mathrm{C}\right.$, a presión $\left.7.4 \mathrm{MPa}\right)$, además permite una fácil extracción de sus componentes. El proceso de extracción por EAFS, consiste en colocar el material vegetal en un reactor, que a su vez va colocado en el recipiente de extracción. Posteriormente, el fluido supercrítico (FS) es cargado desde la parte inferior del recipiente extractor, y al final, el FS que contiene los compuestos extraídos emigra a través de una válvula de despresurización a otra sección del sistema que se llama separador; es ahí donde el extracto se separa del fluido gaseoso debido a que hay una menor presión en el sistema. El extracto es recogido en otra sección llamada colector (Yousefi et al., 2019). Este es un método de extracción que presenta muchas ventajas y permite incrementar los rendimientos de los compuestos extraídos. Ferrentino, Morozova, Mosibo, Ramezani \& Scampicchio (2018) compararon la EAFS con el método soxhlet y la maceración para la obtención de antioxidantes de la pulpa de manzana, en fresco y liofilizada, y los resultados mostraron que los extractos obtenidos por EAFS de la pulpa liofilizada presentaron alta actividad antioxidante $(5.63 \pm 0.10$ TEA/g extracto) comparada con el método convencional soxhlet $(2.05 \pm 0.21 \mathrm{TEA} / \mathrm{g}$ extracto) y el método de maceración con agua en ebullición $(1.14 \pm 0.01 \mathrm{TEA} / \mathrm{g}$ extracto $)$.

Por lo tanto, EAFS es un proceso factible para la recuperación de compuestos bioactivos de productos naturales, ya que aparte de ser una tecnología amigable con el medio ambiente, no daña la salud del usuario, debido a que el uso del $\mathrm{CO}_{2}$ posee una alta seguridad para la salud en comparación con los métodos convencionales.

\section{Conclusiones}

Los residuos generados a partir de los cítricos, en general, ofrecen una buena alternativa para la extracción de compuestos bioactivos con propiedades de interés para las industrias farmacéutica, cosmética y alimentaria. Se ha comprobado que la cáscara posee en mayor proporción compuestos fenólicos, polisacáridos, aceites esenciales y demás compuestos que presentan potencial antimicrobiano y antioxidante. La extracción por métodos alternativos juega un papel relevante por las ventajas que posee, para aplicarse a escala industrial. Por esto, la extracción asistida por ultrasonido es la más estudiada y ofrece mejores rendimientos que las técnicas convencionales, incluso que la maceración, e impacta notablemente en la disminución de la contaminación al medio ambiente, por el bajo consumo de disolventes orgánicos y de gasto energético. Aunado a esto, también es necesario, el aprovechamiento integral de los residuos agroindustriales generados a nivel mundial, con el propósito de brindarles un valor agregado y disminuir la contaminación ambiental generada por la acumulación de este tipo de materiales.

\section{CONFLICTO DE INTERESES}

Los autores declaran no tener conflicto de intereses.

\section{Agradecimientos}

Los autores agradecen al Tecnológico Nacional de México por el financiamiento otorgado para la realización del proyecto con clave 6693.18-P. También se agradece al BQ. José Guadalupe Ávila Hernández por el apoyo técnico.

\section{REFERENCIAS}

Al-juhaimi, F. Y. (2014). Citrus fruits by-products as sources of bioactive compounds with antioxidant potential. Pakistan Journal of Botany, 46 (4), 1459-1462.

Al-Mamoori, F. \& Al-Janabi, R. (2018). Recent advances in microwave-assisted extraction (MAE) of medicinal plants: a review. International Research Journal of Pharmacy, 9(6), 22-29. DOI: 10.7897/2230-8407.09684

Ali, J., Das, B. \& Saikia, T. (2017). Antimicrobial activity of lemon peel (Citrus limon) extract. International Journal of Current Pharmaceutical Research, 9(4), 79-82. DOI: 10.22159/ijcpr.2017v9i4.20962

Alias, N. H. \& Abbas, Z. (2017). Microwave-assisted extraction of phenolic compound from pineapple skins: the optimum operating condition and comparision with soxhlet extraction. Malaysian Journal of Analytical Sciences, 21(3), 690-699. DOI: $10.17576 / \mathrm{mjas}-2017-2103-18$

Ameer, K., Shahbaz, H. M. \& Kwon, J. (2017). Green extraction methods for polyphenols from plant matrices and their by products: a Review. Comprehensive Reviews in Food Science and Food Safety, 16, 295-315. https://doi. org/10.1111/1541-4337.12253

Bandar, H., Hijazi, A., Rammal, H., Hachem, A., Saad, Z. \& Badran, B. (2013). Techniques for the Extraction of Bioactive Compounds from Lebanese Urtica dioica. American Journal of Phytomedicine and Clinical Therapeutics, 1(6), 507-513.

Bermejo, A., Llosá, M. J. \& Cano, A. (2011). Analysis of bioactive compounds in seven Citrus cultivars. Food 
Science and Technology International, 17(1), 55. DOI: 10.1177/1082013210368556.

Boudhrioua, M. N., M'hiri, N., Ioannou, I., Paris, C. \& Ghoul, M. (2016). Comparison of the efficiency of different extraction methods on antioxidants of maltease orange peel. International Journal of Food and Nutritional Science, 3(2), 1-13. DOI: 10.15436/2377-0619.16.789

Bull, O. S. \& Obunwo, C. C. (2014). Bio-diesel production from oil of orange (Citrus sinensis) peels as feedstock. Journal of Applied Sciences and Environmental Management, 18(3), 371-374. DOI: 10.4314/jasem.v18i3.2

Caputo, L., Quintieri, L., Cavalluzzi, M., Lentini, G. \& Habtemariam, S. (2018). Antimicrobial and antibiofilm activities of citrus water-extracts obtained by microwaveassisted and conventional methods. Biomedicines, 6(2), 70. DOI: 10.3390/biomedicines6020070.

Carciochi, R. A., D’Alessandro, L. G., Vauchel, P., Rodriguez, M. M., Nolasco, S. M. \& Dimitrov, K. (2017). Valorization of agrifood by-products by extraction valuable bioactive compounds using green processes. In A. M. Grumezescu \& A. M. Holban (Eds.), Ingredients extraction by physicochemical methods in food (pp. 191-228). London, United Kindgdom:Academic Press. https://doi.org/10.1016/ B978-0-12-811521-3.00004-1

Chavan, P., Singh, A. K. \& Kaur, G. (2018). Recent progress in the utilization of industrial waste and by-products of citrus fruits : A review. Journal of Food Process Engineering, 41, e12895. https://doi.org/10.1111/jfpe.12895. ¿no tiene número de páginas?

Dahmoune, F., Moussi, K., Remini, H., Belbahi, A., Aoun, O., Spigno, G. \& Madani, K. (2014). Optimization of ultrasound-assisted extraction of phenolic compounds from Citrus sinensis L. peels using response surface methodology. Chemical Engineering Transactions, 37, 889-894. DOI: 10.3303/CET1437149.

Damián-Reyna, A. A., González-Hernández, J. C. \& ChávezParga, M. del C. (2016). Procedimientos actuales para la extracción y purificación de flavonoides cítricos. Revista Colombiana de Biotecnología, 18(1), 135-147. DOI: https:// doi.org/10.15446/rev.colomb.biote.v18n1.57724.

Dar, N. G., Hussain, A., Paracha, G. M. \& Akhter, S. (2015). Evaluation of different techniques for extraction of antioxidants as bioactive compounds from Citrus peels (industrial by products). American-Eurasian Journal of Agricultural and Environmental Sciences, 15(4), 676-682.

De-la-Rosa-Hernández, M., Wong-Paz, J. E., Muñiz-Márquez, D. B., Carrillo-Inungaray, M. L. \& Sánchez-González, J. M. (2016). Compuestos fenólicos bioactivos de la toronja (Citrus paradisi) y su importancia en la industria farmacéutica y alimentaria. Revista Mexicana de Ciencias Farmacéuticas, 47(2), 22-35.

Ergüt, M. \& Döker, O. (2017). Recovery of bioactive phenolic compounds from lemon (Citrus limon (L.) Burm .f.) and orange (Citrus sinensis L . Osbeck) Pomaces. Chemical and Process Engineering Research, 51, 18-33.

Ferrentino, G., Morozova, K., Mosibo, O. K., Ramezani, M. \& Scampicchio, M. (2018). Biorecovery of antioxidants from apple pomace by supercritical fluid extraction. Journal of Cleaner Production, 186, 253-261. https://doi. org/10.1016/j.jclepro.2018.03.165

Hernández, M., Ventura, J., Castro, C., Boone, V., Rojas, R., Ascacio-Valdés, J. \& Martínez-Ávila, G. (2018). UPLCESI-QTOF-MS2 -Based identification and antioxidant activity assessment of phenolic compounds from red corn cob (Zea mays L.). Molecules, 23(1425), 1-10.

Hernawan, I., Radithia, D., Hadi, P. \& Ernawati, D. S. (2015). Fungal inhibitory effect of Citrus limon peel essential oil on Candida albicans. Dental Journal (Majalah Kedokteran Gigi), 48(2), 84-88. http://dx.doi.org/10.20473/j.djmkg. v48.i2.p84-88

Khan, M. K., Abert-vian, M., Fabiano-Tixier, A. S., Dangles, O. \& Chemat, F. (2010). Ultrasound-assisted extraction of polyphenols (flavanone glycosides) from orange (Citrus sinensis L.) peel. Food Chemistry, 119(2), 851-858. https:// doi.org/10.1016/j.foodchem.2009.08.046

Kim, J. H. \& Kim, M. Y.(2016). The potential use of Citrus juice waste as sources of natural phenolic antioxidants. Journal of Applied Pharmaceutical Science, 6(12), 202-205. DOI: 10.7324/JAPS.2016.601230

Kodagoda, K. \& Marapana, R. (2017). Utilization of fruit processing by-products for industrial applications: a review. International journal of Food Science and Nutrition, 2(6), 24-30. https://doi.org/10.22271/food

Londoño-Londoño, J., de-Lima, R. V, Lara, O., Gil, A., Pasa, T. C., Arango, G. J. \& Ramírez-Pineda, J. (2010). Clean recovery of antioxidant flavonoids from Citrus peel: optimizing an aqueous ultrasound-assisted extraction method. Food Chemistry, 119(1), 81-87. https://doi. org/10.1016/j.foodchem.2009.05.075

Madhuri, S., Hegde, A. U., Srilakshimi, N. S. \& Prashith, K. (2014). Antimicrobial activity of Citrus sinensis and Citrus aurantium peel extracts. Journal of Pharmaceutical and Scientific Innovation (JPSI), 3(4), 366-368. DOI: 10.7897/2277-4572.034174

Mahato, N., Sharma, K., Sinha, M. \& Cho, M. H. (2018). Citrus waste derived nutra-/pharmaceuticals for health benefits : Current trends and future perspectives. Journal of FunctionalFoods, 40, 307-316. https://doi.org/10.1016/j. jff.2017.11.015

Marín-Rangel, V. M., Cortés-Martínez, R., Cuevas-Villanueva, R. A., Garnica-Romo, M. G., \& Martínez-Flores, H. E. (2012). As (V) biosorption in an aqueous solution using chemically treated lemon (Citrus aurantifolia Swingle) residues. Journal of Food Science, 71(1), 10-14. DOI: 10.1111/j.1750-3841.2011.02466.x.

Martínez, A,. Gutiérrez, S. \& Tlenkopatchev, M. A. (2012). Metathesis transformations of natural products: Crossmetathesis of natural rubber and mandarin oil by 
Ru-alkylidene catalysts. Molecules, 17, 6001-6010. DOI:10.3390/molecules 17056001

Medina-Torres, N., Ayora-Talavera, T., Espinosa-Andrews, H., Sánchez-Contreras, A. \& Pacheco, N. (2017). Ultrasound assisted extraction for the recovery of phenolic compounds from vegetable sources. Agronomy, 7(47), 1-19. https://doi. org/10.3390/agronomy 7030047

Mahato, N., Sharma, K., Sinha, M., Baral, E. R., Koteswararao, R., Dhyani, A., Cho, M. H. \& Cho, S. (2020). Bio-sorbents, industrially important chemicals and novel materials from Citrus processing waste as a sustainable and renewable bioresource: A review. Journal of Advanced Research, 23, 61-82. https://doi.org/10.1016/j.jare.2020.01.007

Muñiz-Márquez, D. B., Rodríguez, R., Balangurusamy, N., Carrillo, M. L., Belmares, R., Contreras, J. C. \& Aguilar, C. N. (2013a). Phenolic content and antioxidant capacity of extracts of Laurus nobilis L ., Coriandrum sativum L. and Amaranthus hybridus L . CyTA - Journal of Food: 12(3), 271-276. https://doi.org/10.1080/19476337.2013.847500

Muñiz-Márquez, D. B., Martínez-Ávila, G. C., Wong-Paz, J. E., Belmares-Cerda, R., Rodríguez-Herrera, R. \& Aguilar, C. N. (2013b). Ultrasound-assisted extraction of phenolic compounds from Laurus nobilis L. and their antioxidant activity. Ultrasonics Sonochemistry, 20, 1149-1154. https:// doi.org/10.1016/j.ultsonch.2013.02.008

Nakajima, V. M., Macedo, G.A. \& Macedo, J. A. (2014). Citrus bioactive phenolics: role in the obesity treatment. $L W T$ Food Science and Technology, 59, 1205-1212. https://doi. org/10.1016/j.lwt.2014.02.060

Nayak, B., Dahmoune, F., Moussi, K., Remini, H., Dairi, S., Aoun, O. \& Khodir, M. (2015). Comparison of microwave, ultrasound and accelerated-assisted solvent extraction for recovery of polyphenols from Citrus sinensis peels. Food Chemistry, 187, 507-516. https://doi.org/10.1016/j. foodchem.2015.04.081

Pathak, P. D., Mandavgane, S. A. \& Kulkarni, B. D. (2015). Fruit peel waste as a novel low-cost bio adsorbent. Reviews in Chemical Engineering, 31(4), 361-381. DOI: 10.1515/ revce-2014-0041

Putnik, P., Bursać-Kovačević, D., Režek-Jambrak, A., Barba, F., Cravotto, G., Binello, A. \& Shpigelman, A. (2017). Innovative "green" and novel strategies for the extraction of bioactive added value compounds from Citrus wastes - a review. Molecules, 22(5), 680. DOI: 10.3390/ molecules22050680.

Rafiq, S., Kaul, R., Sofi, S. A., Bashir, N., Nazir, F. \& Nayik, A. G. (2018). Citrus peel as a source of functional ingredient: a review. Journal of the Saudi Society of Agricultural Sciences, 17, 351-358. https://doi.org/10.1016/j. jssas.2016.07.006

Sadeghi,A., Hakimzadeh, V. \& Karimifar, B. (2017). Microwave assisted extraction of bioactive compounds from food: a review. International Journal of Food Science and Nutrition Engineering, 7(1), 19-27.DOI:10.5923/j.food.20170701.03
Safdar, M. N., Kausar, T., Jabbar, S., Mumtaz, A., Ahad, K. \& Saddozai, A. A. (2017). Extraction and quantification of polyphenols from kinnow (Citrus reticulate L.) peel using ultrasound and maceration techniques. Journal of Food and Drug Analysis, 25, 488-500. https://doi.org/10.1016/j. jfda.2016.07.010

Sánchez-Aldana, D., Aguilar, C. N., Nevarez-Moorillon, G. V. \& Esquivel-Contreras, J.C. (2013). Comparative extraction of pectin and polyphenols from mexican lime pomace and bagasse. American Journal of Agricultural and Biological Science, 8(4),309-322. DOI: 10.3844/ajabssp.2013.309.322

Selvamuthukumaran, M. \& Shi, J. (2017). Recent advances in extraction of antioxidants from plant by-products processing industries. Food Quality and Safety, 1(1), 61-81. https:// doi.org/10.1093/fqsafe/fyx004

Shie, P. H. and \& Lay, H. L. (2013). Component analysis and antioxidant activity of Citrus limon. Academia Journal of Medicinal Plants, 1(3), 49-58.

Soquetta, M. B., Terra, L. D. M. \& Bastos, C. P. (2018). Green technologies for the extraction of bioactive compounds in fruits and vegetables. CyTA - Journal of Food, 16(1), 400-412. https://doi.org/10.1080/19476337.2017.1411978

Tsatsop, R. K. T., Djiobie, G. T., Kenmogne, B. S., Regonne, K. R. \& Ngassoum, M. B. (2016). Optimization of microwave-assisted extraction of bioactive compounds from Anogeissus leiocarpus Guill. \& Perr. stem bark using response surface methodology. International Journal of Scientific \& Technology Research, 5(5), 103-112.

Tumbas, V.T., Ćetković, G. S.\&Djilas, S. M.(2010). Antioxidant activity of mandarin (Citrus reticulata) peel. APTEFF, 41(1), 195-203. DOI: 10.2298/APT1041195T

Vergamini, D., Cuming, D. \& Viaggi, D. (2015). The integrated management of food processing waste : the use of the full cost method for planning and pricing mediterranean citrus by-products. International Food and Agribussines Management Review, 18(2), 153-172. DOI: 10.22004/ ag.econ.204141

Wang, L. \& Weller, C. L. (2006). Recent advances in extraction of nutra- ceuticals from plants. Trends in Food Science and Technology, 17, 300-312. https://doi.org/10.1016/j. tifs.2005.12.004

Wong-Paz, J. E., Muñiz-Márquez, D., Aguilar-Zarate, P., Ascacio-Valdés, J., Cruz, K., Reyes-Luna, C. \& Aguilar, C. (2017). Extraction of Bioactive Phenolic Compounds by Alternative Technologies. In A. M. Grumezescu \& A. M. Holban (Eds.), Ingredients Extraction by Physicochemical Methods in Food (pp. 229-252). London, United Kindgdom: Academic Press. DOI: 10.1016/B9780-12-811521-3.00005-3

Wong-Paz, J. E., Muñiz-Márquez, D. B., Martínez-Ávila, G. C. G., Belmares-Cerda, R. E. \& Aguilar, C. N. (2015). Ultrasound-assisted extraction of polyphenols from native plants in the Mexican desert. Ultrasonics Sonochemistry, 22, 474-481. https://doi.org/10.1016/j.ultsonch.2014.06.001 
Wu, T., Guan, Y. \& Ye, J. (2007). Determination of flavonoids and ascorbic acid in grapefruit peel and juice by capillary electrophoresis with electrochemical detection. Food Chemistry, 100, 1573-1579. https://doi.org/10.1016/j. foodchem.2005.12.042

Yousefi, M., Nasrabadi, M. R., Pourmortazavi, S. M., Wysokowski, M., Jesionowski, T., Ehrlich, H. \& Mirsadeghi, S. (2019). Supercritical fluid extraction of essential oils.
Trends in Analytical Chemistry, 118, 182-193. https://doi. org/10.1016/j.trac.2019.05.038

Zhao, S., Ren, W., Gao, W., Tian, G., Zhao, C., Bao, Y., Cui, J., Lian, Y. \& Zheng J. (2020). Effect of mesoscopic structure of citrus pectin on its emulsifying properties: compactness is more important than size. Journal of Colloid and Interface Science, 570, 80-88. https://doi.org/10.1016/j. jcis.2020.02.113. 